\title{
EFECTOS DE LA CONTAMINACIÓN ATMOSFÉRICA SOBRE LA SALUD: UNA INTRODUCCIÓN
}

\author{
Ferran Ballester Díez (1), José María Tenías (1,2) y Santiago Pérez-Hoyos (1) \\ (1) Institut Valencià d'Fstudis en Salut Pública (IVESP). Dirección General de Salud Pública. Conselleria de Sanitat. \\ Generalitat Valenciana. \\ (2) Hospital Lluís Alcanyís. Xàtiva. Conselleria de Sanitat. Generalitat Valenciana.
}

\section{RESUMEN}

En los últimos años ha habido un gran avance en el conocimiento y comprensión de los efectos de la contaminación atmosférica. En este trabajo se establecen sus conceptos fundamentales y se revisan las diferentes aproximaciones metodológicas posibles. Entre los estudios epidemiológicos, los de series temporales son los más utilizados para medir los efectos a corto plazo de la contaminación atmosférica. Los factores de confusión más importantes en este tipo de estudios son las variaciones estacionales y semanales, la tendencia, las variables meteorológicas y las enfermedades graves con un comportamiento estacional. como la gripe.

Los principales efectos a corto plazo de la contaminación atmosférica sobre la salud vari desde ur aumento de la mortalidad total y por causas respiratorias y cardiovasculares a las alteraciones del funcionalismo pulmonar y otros sintomas, pasando por un incremento en el número de visitas médicas e ingresos hospitalarios.

A pesar de existir un amplio consenso en cuanto al efecto nocivo de la contaminación atmosférica, existen una serie de cuestiones que necesitan de mayor investigación.

Palabras clave: Contaminación atmosférica. Efectos en salud. Series temporales.

\section{INTRODUCCIÓN}

La atmósfera es la parte del ambiente con la que el organismo humano está permanentemente en contacto. De hecho, muchas de

Correspondencia:

Ferran Ballester Díez

Institut Valencià d'Estudis en Salut Pública (IVESP).

Juan de Garay, 21.

46017 Valencia.

Teléfono 96-3869369

Fax 96-3869370

Correo electrónico: fballest@san.gva.es

\section{ABSTRACT}

\section{The Impact of Air Pollution on Human Health: An Introduction}

In recent years, major progress has been made as regards the knowledge and understanding which has been gained of the impact of air pollution. In this study, the basic concepts are set out regarding this subject, and the different possible approaches as far as methodologies are concerned are reviewed. Among the epidemiological studies, the time series studies are those most often used for assesing the short-term impact of air pollution. The most important factors leading to confusion in this type of study are the seasonal and weekly changes, the tendency, the weather variables and the serious illnesses of a seasonal pattern, such as the flu.

The main short-term impact of air pollution on human health range from a rise in the overall death rate resulting from respiratory and cardiovascular diseases to the worsening of lung function and other symptoms, including a rise in the number of doctor visits and hospital admissions.

Despite a widespread consensus existing with regard to the harmful impact of air pollution, there are a number of questions currently in the need of further rescarch.

Key words: Air pollution. Impact on human health. Time series studies. sus reacciones pueden ser explicadas como la respuesta a los cambios en el estado físico y químico de la atmósfera ${ }^{1}$. Tanto en el campo de la clínica como en el de la salud pública, la contaminación atmosférica es un fenómeno conocido y estudiado desde antiguo. En el mundo contemporáneo cobra una gran importancia a partir de una serie de episodios que tuvieron lugar en los países industrializados durante la primera mitad del siglo XX. Los casos ocurridos en el Valle de Mosa (Bélgica) en 1930, en Donora (Pennsylvania, EEUU) en 1948 y, sobre todo, la 
catástrofe de Londres, en Diciembre de 1952, serían tal vez los más destacables y característicos $^{2,3}$. Estas situaciones excepcionales se tradujeron en un aumento de la mortalidad y la morbilidad, que no dejaron dudas acerca de que los niveles altos de contaminación atmosférica se asocian causalmente con un aumento de muertes tempranas. En el último de los episodios citados, una densa niebla cubrió el área del Gran Londres durante 4 días, del 5 al 8 de Diciembre de 1952, acompañándose de un brusco aumento en la mortalidad. El número de muertes en exceso atribuidas a este episodio fue entre 3.500 y 4.000 . Estas evidencias llevaron a la adopción de políticas de control de la contaminación, especialmente en Europa Occidental y en los Estados Unidos, las cuales han conducido a una importante reducción de los niveles de contaminación atmosférica.

Aunque los niveles actuales de contaminación atmosférica en los países del mundo occidental pueden, en general, considerarse moderados, la preocupación acerca de sus posibles efectos en la salud de las personas persiste. Por un lado, en los últimos años un número importante de estudios realizados en distintas ciudades ha encontrado que, aún por debajo de los niveles de calidad del aire considerados como seguros, los incrementos de los niveles de la contaminación atmosférica se asocian con efectos nocivos sobre la salud ${ }^{4.5}$. Por otro lado, aumenta la preocupación sobre los posibles riesgos de agentes para los que no existe una evaluación satisfactoria ${ }^{6}$. Importantes sectores de la población se encuentran expuestos a contaminantes atmosféricos con posibles repercusiones negativas sobre su salud. En respuesta a las demandas de la sociedad, tanto los médicos clínicos, como los toxicólogos y epidemiólogos, están llamados a evaluar los efectos adversos de los contaminantes inhalados: el clínico evaluando la salud de los individuos expuestos, el toxicólogo definiendo el daño causado por el contaminante, y el epidemiólogo estudiando los efectos en los grupos expuestos. Todos estos tipos de valoración son complementarios, dado que cada uno tiene ventajas y limitaciones para dar respuesta a las preocupaciones de la sociedad ${ }^{6}$.

El objetivo de este artículo es servir de introducción al número monográfico sobre los efectos de la contaminación atmosférica. Para ello se proporciona una aproximación a los principales métodos utilizados, se consideran las variables de confusión más importantes y se indican los efectos, de los que existe mayor evidencia, de la contaminación atmosférica en la salud.

\section{APROXIMACIONES METODOLÓGICAS}

La interpretación de las reacciones que produce la contaminación atmosférica en la salud humana se fundamenta en estudios de dos clases, toxicológicos y epidemiológicos. Ambos tipos de estudios se consideran complementarios cuando se trata de valorar los efectos de la contaminación atmosférica en la salud.

\section{- Estudios toxicológicos experimen-} tales, en el hombre o en los animales, en los que la concentración, duración y condiciones de la exposición son controladas por el investigador ${ }^{7}$. Su principal ventaja radica precisamente en el control de las condiciones de exposición, por lo que la medida de ésta es más precisa que en el caso de los estudios observacionales. En cuanto a los inconvenientes, éstos radican fundamentalmente en el hecho de someter a los sujetos en experimentación a una situación artificial, ya que se utiliza un reducido número de contaminantes en situación ideal, a grandes dosis y en circunstancias ambientales especiales. Además, las poblaciones con las que se experimenta son poco representativas.

- Estudios epidemiológicos, en los que se observan los sucesos que se desarrollan en las poblaciones humanas bajo condiciones 
naturales, circunstancia en la que radica su ventaja más significativa. Como medida de la contaminación atmosférica se utilizan, habitualmente, los datos de las redes de vigilancia de la contaminación atmosférica. En este caso el principal problema es la medida de la exposición, ya que el estudio epidemiológico de los efectos sobre la salud de la contaminación atmosférica tiene una serie de características que lo distinguen de otras áreas de la epidemiología. Entre éstas destacaríamos ${ }^{8}$ :

a. Ubicuidad de la exposición: una vez los contaminantes han sido vertidos al aire, $o$ se han formado en él, la exposición no se puede evitar. Esto no ocurre igual en otros medios, como el agua de bebida, el suelo o los alimentos. Por este motivo, es dificil encontrar grupos no expuestos en los estudios epidemiológicos. Sin embargo, a pesar de su ubicuidad la contaminación atmosférica no se distribuye de manera homogénea en las ciudades.

b. Variaciones individuales de la exposición: incluso cuando las concentraciones de los contaminantes en el ambiente exterior son más o menos uniformes (lo cual raramente ocurre), los individuos pasamos la mayor parte del tiempo en nuestras casas y en el interior de otros lugares. La exposición del ambiente exterior puede diferir considerablemente de la del interior, debido a la presencia de fuentes interiores, a la deposición y a las diferencias en la ventilación. Sin embargo, allí donde no existen fuentes interiores significativas, la calidad del aire interior viene determinada, de una manera importante, por las concentraciones en el exterior.

c. Confusión importante por otros factores: la morbilidad y mortalidad por enfermedades respiratorias y del aparato circulatorio pueden tener causas diferentes a la contaminación atmosférica. Además, estos factores, pueden tener una influencia más fuerte, como son el hábito tabáquico o la exposición a gases y polvos irritantes en el ámbito profesional.

\section{LOS ESTUDIOS ECOLÓGICOS DE SERIE TFMPORALES}

Uno de los diseños epidemiológicos más utilizados en la actualidad son los estudios ecológicos de series temporales. En ellos se analizan las variaciones en el tiempo de la exposición al contaminante y el indicador de salud estudiado en una población. Los estudios ecológicos miden exposiciones ambientales y efectos en poblaciones humanas representativas. En ellos se considera todo el rango de exposiciones, así como las múltiples combinaciones de factores ambientales y socioculturales que determinan la ocurrencia de los efectos sobre la salud. Una de las grandes ventajas de los estudios de series temporales es que al analizar a la misma población en diferentes periodos de tiempo (día a día, generalmente), muchas de aquellas variables que pueden actuar como factores de confusión a nivel individual (hábito tabáquico, edad, género, ocupación, etc.) no necesitan ser controladas, ya que se mantienen estables en la misma población. En todo caso, no es previsible que las variaciones que éstas puedan sufrir día a día se relacionen con los niveles de contaminación atmosférica.

La falacia ecológica, el principal sesgo de estos estudios, se define como el error cometido al trasladar las estimaciones realizadas del nivel ecológico a un nivel individual $^{9}$. Generalmente, la falacia ecológica afccta más a los diseños geográfícos o espaciales que a los de series temporales. Por otro lado, y debido a que siempre se trata de mezclas de contaminantes, existe dificultad en atribuir los efectos sobre la salud a un contaminante concreto.

Al analizar los estudios ecológicos de series temporales que examinan la relación entre contaminación atmosférica y salud, se debería tener en cuenta, por un lado, la adecuación de la metodología estadística utilizada $^{10,11} \mathrm{y}$, por otro, los factores de confusión considerados. Estos últimos se pueden resumir en tres grandes grupos ${ }^{12}$ : 
- Factores geofísicos: aquellos producidos por los ciclos estacionales de energía y radiaciones en la superficie de la tierra. Conllevan cambios en la meteorología, la contaminación y la salud. Su ciclo fundamental es el año.

- Factores meteorológicos: aunque podrían incluirse dentro de los factores geofísicos ya que dependen de estos, tienen una entidad propia innegable. La temperatura ambiental y la humedad son dos variables con las que se intenta resumir la meteorología, lo que para algunos autores resulta insuficiente para poder ajustar adecuadamente un fenómeno tan complejo ${ }^{13}$.

- Factores socioculturales: se corresponden con el nivel de desarrollo económico e industrial, la cultura, la reli- gión, la mentalidad de las personas, el país, la comunidad, etc. El ciclo fundamental de la actividad sociocultural en la mayoría de países y culturas es la semana, que se corresponde con el ciclo laboral básico. La salud cambia según el día de la semana (por razones aún no del todo conocidas las personas generalmente nos sentimos peor el lunes que el resto de días de la semana, la mortalidad aumenta los días laborables, etcétera y también lo hacen los niveles de contaminación atmosférica.

La construcción de cualquier modelo matemático que analice la relación entre contaminación atmosférica y salud, debería tener en cuenta estos tres grandes grupos de factores. Además, habría que considerar aquellas enfermedades con comportamiento estacional, como la gripe (figura 1).

Figura 1

Relación jerárquica de los factores implicados en el estudio del efecto de la contaminación atmosférica sobre la salud

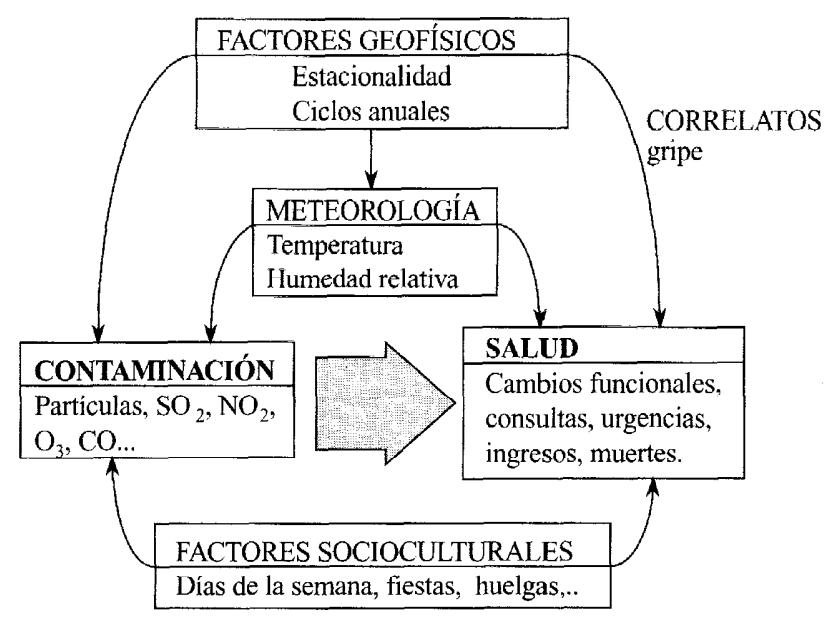

Fuente: Adaptada de Goldsmith et al, $1996^{12}$ 


\section{ESTUDIOS MULTICÉNTRICOS}

En la actualidad existen en marcha diversos proyectos internacionales con criterios de análisis estandarizados para el estudio de diferentes aspectos de la relación entre la contaminación atmosférica y la salud. En Estados Unidos, el Instituto para los efectos de la salud (HEI), corporación no lucrativa patrocinada por la agencia de protección ambiental americana (EPA), tiene como principal objetivo estudiar los efectos sobre la salud de los contaminantes atmosféricos emitidos por los vehículos a motor y otras fuentes. Entre sus últimos proyectos se encuentra el reanálisis de los estudios de cohortes que investigan la influencia de la polución aérea por partículas sobre la mortalidad a largo plazo ${ }^{14}$.

En Europa, el proyecto $A P H E A$ (Short term effects of air pollution on health, a European approach) es uno de los más ambiciosos de cuantos se están realizando. Este estudio multicéntrico contó en su primera fase con la participación de 15 ciudades europeas (entre las que se incluía Barcelona) pertenecientes a 10 países diferentes, con una población de estudio de aproximadamente 25 millones de habitantes ${ }^{15}$. En la segunda fase del proyecto, que incluye $34 \mathrm{ciu}-$ dades, además de Barcelona participan investigadores de Madrid, Bilbao y Valencia $^{16}$. En el ámbito español, el proyecto EMECAM (Estudio Multicéntrico Español sobre la relación entre la Contaminación Atmosférica y la Mortalidad), se enmarca dentro del contexto de los estudios multicéntricos citados. En dicho proyecto se incluyen 14 ciudades españolas (Barcelona, Gran Bilbao, Cartagena, Castellón, Gijón, Huelva, Madrid, Pamplona, Sevilla, Oviedo, Valencia, Vigo, Vitoria y Zaragoza) que representan diferentes situaciones sociodemográficas, climáticas y ambientales, sumando un total de cerca de 9 millones de habitantes.

En otro estudio multicéntrico europeo (Pollution Effects on Asthmatic Children in
Europe, $P E A C E)^{17}$ se estudian los efectos agudos de la contaminación atmosférica causada por partículas, $\mathrm{SO}_{2}$ y $\mathrm{NO}_{2}$ en la salud respiratoria de 2010 niños repartidos en 28 paneles de otras tantas localidades europeas. Los objetivos de dicho estudio son obtener datos comparables en las concentraciones de partículas durante el periodo invernal, en varias localidades urbanas y no urbanas de Europa, valorar la relación entre las fluctuaciones a corto plazo en la contaminación atmosférica y las fluctuaciones a corto plazo en los indicadores de salud respiratoria de los niños con sintomatología respiratoria crónica, evaluar si las características médicas de los niños están relacionadas con las diferencias en la respuesta a la contaminación atmosférica $y$, por último, evaluar si la composición de las partículas se relaciona con la respuesta a la contaminación atmosférica.

\section{EFECTOS DE LA CONTAMINACIÓN ATMOSFÉRICA SOBRE LA SALUD}

Los efectos relacionados con la exposición a la contaminación atmosférica son diversos. Los más estudiados son aquellos que se producen a corto plazo, es decir en el periodo de unos pocos días, habitualmente menos de una semana, después de la exposición (tabla 1). Estos efectos mantienen una gradación tanto en la gravedad de sus consecuencias como en la población a riesgo afectada (figura 2). Además, deben estar relacionados por el «principio de coherencia») definido por Bates ${ }^{18}$. Por ejemplo, si el hallazgo principal es un aumento de la mortalidad total o por una causa específica, se debería esperar, necesariamente, salvo que todos los que mueren en exceso ya estén hospitalizados, un incremento en los ingresos hospitalarios. A este efecto se le conoce como hallazgo contingente. Si el hallazgo principal es un aumento en el número de ingresos hospitalarios, debcríamos encontrar, como efecto contingente, un incremento paralelo del número de visitas a urgencias. Este principio de coherencia es difícil de 
Tabla 1

Principales efectos a corto plazo de la contaminación atmosférica sobre diferentes indicadores de salud

\begin{tabular}{|c|}
\hline $\begin{array}{c}\text { Aumento de la mortalidad total y por causas especificas } \\
\text { Incremento de la utilización de los servicios sanitarios } \\
\text { Ingresos hospitalarios } \\
\text { Visitas a urgencias } \\
\text { Visitas a consultas médicas } \\
\text { Alteraciones de diferentes índices funcionales pulmonares } \\
\text { Incremento de los síntomas de enfermedad y del uso de fármacos }\end{array}$ \\
\hline
\end{tabular}

Fuente: A Committe of the Envimnmental and Occupational Health Assembly of the American Thoracic Society, $1996^{6}$

Figura 2

Representación de los diferentes efectos de la contaminación atmosférica sobre la salud

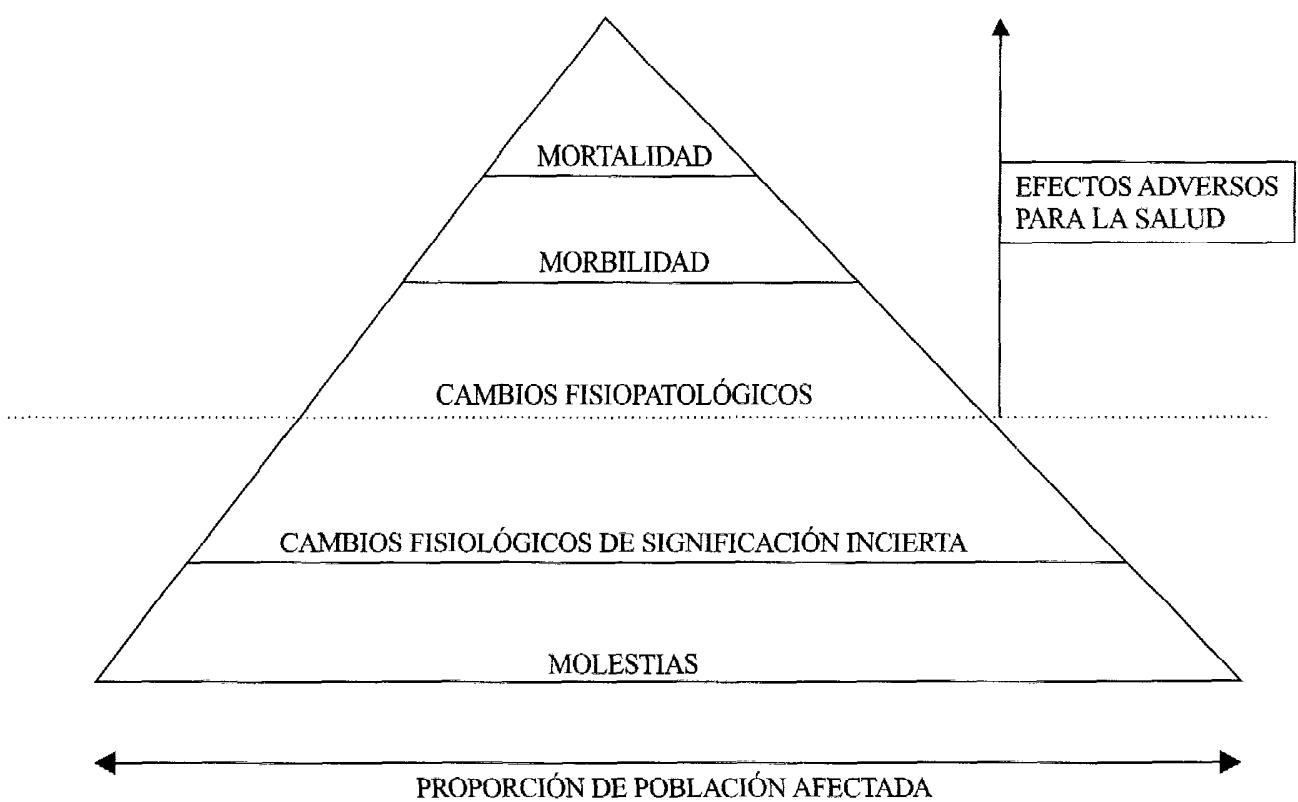

Fuente: Traducida y adaptada de Andrews et al, $1985^{42}$

comprobar, ya que para demostrarlo en su totalidad deberían verificarse todos los hallazgos principales y sus efectos contingentes en la misma localización geográfica y en el mismo periodo de tiempo, pues el lugar y el tiempo pueden actuar como determinantes o, al menos, matizar en gran medida los resultados.
A la hora de hablar de los efectos de la contaminación atmosférica sobre la salud es conveniente diferenciar entre la contaminación más típica del invierno (winter smog) y la que es más característica del verano (summer smog). En invierno los episodios de contaminación pueden ocurrir debido a condiciones de estancamiento del aire, cuando 
los contaminantes procedentes de la combustión se acumulan en la atmósfera. Los contaminantes principales son el $\mathrm{SO}_{2}$ y las partículas en suspensión, aunque éstos simplemente sirven como indicadores de mezclas de contaminantes mucho más complejas. En verano los episodios de contaminación pueden ocurrir en los días calurosos y soleados, en los que las reacciones fotoquímicas de los óxidos de nitrógeno y los hidrocarburos llevan a la formación de ozono y otras sustancias con capacidad tóxica ${ }^{19}$.

\section{Efectos de la contaminación tipo invierno («winter smog»)}

Los componentes principales de dicho tipo de contaminación son el $\mathrm{SO}_{2}$ y las partículas en suspensión. $\mathrm{El} \mathrm{SO}_{2}$ es un gas irritante que a altas concentraciones en individuos normales y más bajas en individuos asmáticos, puede producir broncoconstricción. En 1987, la Oficina Regional de la Organización Mundial de la Salud (OMS) para Europa estableció $1000 \mu \mathrm{g} / \mathrm{m}^{3}$ como el nivel más bajo al cual se observarían efectos después de 10 minutos de exposición en individuos asmáti$\cos ^{20}$. Aplicando un factor corrector de 2 , resulta un nivel de $500 \mu \mathrm{g} / \mathrm{m}^{3}$ como nivel guía de protección de la Salud Pública para «valores pico». Debería tenerse en cuenta que esta guía está pensada para proteger la salud de los efectos del $\mathrm{SO}_{2}$, pero no tiene en cuenta la presencia de otras sustancias peligrosas en el aire. Por ello, como la contaminación por $\mathrm{SO}_{2}$ comúnmente se asocia a la de las partículas, sus efectos han sido estudiados en conjunto.

Los efectos agudos del $\mathrm{SO}_{2}$ y las partículas se han examinado en relación con los cambios diarios en mortalidad en un número importante de ciudades, en relación con ingresos y urgencias hospitalarias, con cxacerbaciones de enfermedades en grupos sensibles de sujetos, o con cambios temporales del funcionalismo pulmonar entre niños o adultos. Los efectos agudos esperados en la salud humana establecidos por la OMS en $1990^{21}$, en función de las observaciones hechas en estudios epidemiológicos para niveles promedio de 24 horas, se muestran en la tabla 2 .

En la última década, sin embargo, un importante número de estudios ha puesto en cuestión los criterios mencionados arriba. En buena parte de ellos se informa la asociación de los niveles de contaminación atmos-

Tabla 2

Niveles promedio de 24 horas de mezclas de contaminación atmosférica conteniendo $\mathrm{SO}_{2}$ y partículas en suspensión por encima de los cuales se pueden esperar efectos agudos sobre la salud humana según la OMS (1990)

\begin{tabular}{|clll|}
\hline $\mathrm{SO}_{2}{ }^{*}$ & $\begin{array}{c}\text { Particulas } \\
\text { (método de determinación) }\end{array}$ & \multicolumn{1}{c|}{ Efectos sobre la salud } & $\begin{array}{c}\text { Grado de clasificación } \\
\text { de los efectos }\end{array}$ \\
\hline 200 & 200 (gravimetría, PTS) & $\begin{array}{l}\text { Disminución ligera y transitoria de la función } \\
\text { pulmonar (FVC, FEV })^{2} \text { en niños y adultos que } \\
\text { puede durar unas 2-4 semanas }\end{array}$ & moderados \\
\hline 250 & 250 («humos negros») & $\begin{array}{l}\text { Aumento de la morbilidad respiratoria entre los } \\
\text { adultos susceptibles y, posiblemente, entre los } \\
\text { niños }\end{array}$ & moderados \\
\hline 400 & 400 («humos negros») & Mayor incremento en la morbilidad respiratoria & graves \\
\hline 500 & 500 («humos negros») & $\begin{array}{l}\text { Aumento de la mortalidad entre los ancianos y } \\
\text { los enfermos crónicos }\end{array}$ & graves \\
\hline
\end{tabular}

Fuente: OMS (1990) ${ }^{21}$.

Niveles en $\mu \mathrm{g} / \mathrm{m} 3$

FVC: Capacidad vital forzada. FEV : Volumen espirado durante el primer segundo de la FVC. 
férica con efectos sobre la salud, incluso por debajo de los estándares considerados como seguros por las agencias internacionales. Además, especialmente en el caso de las partículas, cuando se examina la relación dosis-respuesta entre los niveles de contaminación y los indicadores de salud, no se encuentra evidencia de un posible umbral por debajo del cual no se esperen efectos adversos. La tabla 3, tomada de un trabajo realizado por Dockery y Pope ${ }^{22}$, sirve como resumen de los efectos agudos de las partículas más frecuentemente puestos en evidencia. Algunas de las observaciones de esta tabla están basadas en estudios que usaron medidas de partículas por el método de los «humos negros» (reflectometría), mientras otras se basaron en medidas gravimétricas (partículas totales en suspensión). En este sentido, los autores consideraron que el valor obtenido por el método de determinación de los «humos negros», método de referencia en la Unión Europea, sería aproximadamente igual al obtenido por el método de captación de las partículas de diámetro inferior a 10 micras $\left(\mathrm{PM}_{10}\right)$, medida de referencia de la Agencia de Protección Ambiental (EPA) de los Estados Unidos de América. Ambas medidas equivaldrían, aproximadamente, según estos autores, al $55 \%$ de las partículas totales en suspensión (PTS) ${ }^{22}$. Esta conversión ha sido cuestionada al considerarla muy imprecisa, por no tener en cuenta las variaciones en la relación entre dichos indicadores de partículas, tanto al considerar distintas localidades como a lo largo de las estaciones del año ${ }^{23}$. Así, en el contexto del estudio $P E A C E^{24}$, se encontró que la razón entre las medianas de $\mathrm{PM}_{10} \mathrm{y}$ de «humos negros» (HN) en el periodo invernal varió entre 0,67 y 3,67, según las localidades estudiadas. En algunos lugares, sin embargo, como Atenas, Europa central y Oslo, está razón fue próxima a la unidad. Parece haber una tendencia a razones $\mathrm{PM}_{10}$ ' HN más bajas en las áreas urbanas, consistente con la contribución de emisiones de los motores de los vehículos (diesel).

Tabla 3

Estimación de los efectos asociados a los niveles medios diarios de contaminación atmosférica por particulas en suspensión

\begin{tabular}{|c|c|}
\hline Indicador del efecto sobre la salud & $\begin{array}{l}\text { Cambio (\%) del indicador de efecto asociado } \\
\text { a un incremento de } 10 \mu \mathrm{g} / \mathrm{m}^{3} \text { de } P M_{10}{ }^{*}\end{array}$ \\
\hline \multicolumn{2}{|l|}{ Incremento de la mortalidad diaria } \\
\hline - Muertes por todas las causas & 1,0 \\
\hline - Muertes por causas respiratorias & 3,4 \\
\hline - Muertes por causas del aparato circulatorio & 1,4 \\
\hline \multicolumn{2}{|l|}{$\begin{array}{l}\text { Incremento de la demanda de atención hospitalaria } \\
\text { (enfermedades respiratorias) }\end{array}$} \\
\hline - Ingresos & 0,8 \\
\hline — Urgencias & 1,0 \\
\hline \multicolumn{2}{|l|}{ Exacerbación del asma } \\
\hline - Crisis asmáticas & 3,0 \\
\hline — Uso de broncodilatadores & 2,9 \\
\hline — Visitas a urgencias & 3,4 \\
\hline - Ingresos hospitalarios & 1,9 \\
\hline \multicolumn{2}{|l|}{ Disminución de la función pulmonar } \\
\hline - Volumen expiratorio máximo por segundo & 0,15 \\
\hline - Flujo expiratorio máximo & 0,08 \\
\hline
\end{tabular}

*PM $\mathrm{PM}_{10}$ : partículas de diámetro aerodinámico inferior a $10 \mu \mathrm{m}$

Fuente: Dockery y Pope, $1994^{22}$ 
Al conocimiento proveniente de los estudios epidemiológicos observacionales y a pesar de las limitaciones comentadas anteriormente, en los últimos tres años se han aportado algunas evidencias por parte de los estudios experimentales. Por un lado, se ha sugerido que el poder tóxico de las partículas en los ambientes urbanos procedería de su alta composición en partículas ultrafinas. Así, aunque gravimétricamente su concentración no sea muy alta, estas partículas podrían provocar una exacerbación de los problemas respiratorios e incrementar la coagulabilidad plasmática ${ }^{25}$. En un estudio realizado en Alemania ${ }^{26}$ se encontró que durante un episodio de contaminación atmosférica con niveles elevados de $\mathrm{SO}_{2}$ y de partículas en suspensión, las cifras de viscosidad sanguínea se encontraban elevadas, tanto en hombres como en mujeres. Por otro lado, algunos estudios toxicológicos con animales de experimentación han encontrado que los animales expuestos a partículas finas enferman y, a veces, mueren ${ }^{27}$. En uno de estos estudios, realizado en la Escuela de Salud Pública de Harvard, en Boston, se encontró que las ratas expuestas durante 3 días a partículas de diámetro inferior a 2,5 $\mu \mathrm{m}\left(\mathrm{PM}_{2.5}\right)$, extraídas del aire de Boston durante 3 días, a niveles equivalentes al doble de los valores límites actuales de $\mathrm{PM}_{10}$ para 6 horas en la normativa de la EPA, murieron el $37 \%$ de las bronquíticas y sobrevivieron todas las sanas. En otro experimento llevado a cabo con perros expuestos a niveles de partículas entre 116 y $175 \mu \mathrm{g} / \mathrm{m}^{3}$, niveles que a menudo se alcanzan en las ciudades más contaminadas, los animales desarrollaron arritmias y otros trastornos cardíacos. Todos estos datos podrían ayudar a comprender el efecto de las partículas sobre la salud.

El énfasis dado en los Estados Unidos a los efectos adversos de las partículas y el debate que ello ha generado, ha desencadenado la puesta en marcha de muchos estudios sobre el impacto de este contaminante. El estudio de los efectos independientes del $\mathrm{SO}_{2}$ ha recibido en los últimos años menor atención. Sin embargo, en Europa, gracias a los resultados del proyecto multicéntrico APHEA, disponemos de estimaciones de los efectos sobre la salud de este contaminante, obtenidas en estudios recientes y que utilizan metodología adecuada para el análisis de series temporales ${ }^{15}$. El resultado combinado para las 12 ciudades del proyecto APHEA indica un incremento del $4 \%$ en la mortalidad por incremento de $100 \mu \mathrm{g} / \mathrm{m}^{3}$ en los niveles medios diarios de $\mathrm{SO}_{2}{ }^{28}$.

Es posible que estos dos contaminantes, partículas en suspensión y $\mathrm{SO}_{2}$, desempeñen un complejo papel en el desarrollo de enfermedades crónicas. Se ha informado la existencia de asociación entre los niveles de partículas y una disminución del funcionalismo pulmonar ${ }^{29,30}$. Por otro lado, se han llevado a cabo diversas investigaciones sobre la asociación de la contaminación atmosférica y la mortalidad a largo plazo. Existen dos estudios prospectivos de cohortes muy importantes. En el primero, conocido como el estudio de las seis ciudades ${ }^{14}$, se siguen, desde 1974 , a 8.111 adultos de 6 ciudades de los Estados Unidos. Sus resultados indican que las tasas de mortalidad están asociadas con la contaminación del aire, una vez controlado por el hábito de fumar y otros factores de riesgo. El riesgo de morir en las ciudades más contaminadas fue un $26 \%$ más alto comparado con las menos contaminadas. En el segundo estudio Pope et al ${ }^{31}$ evaluaron los efectos de la contaminación atmosférica por partículas sobre la mortalidad, utilizando los datos de contaminación de 151 áreas metropolitanas de los Estados Unidos desde 1980 y siguiendo a 552.138 adultos desde 1982 . Se encontró que el riesgo de morir por todas las causas en las áreas más contaminadas era un $15 \%$ más alto que en las menos contaminadas.

\section{Efectos de la contaminación tipo «verano» («summer smog»). Impacto sobre la salud del ozono}

La contaminación tipo «verano» se refiere principalmente a la contaminación foto- 
química procedente de las reacciones de los hidrocarburos y los óxidos de nitrógeno, estimuladas por la luz solar intensa. El ozono es considerado generalmente como el componente más tóxico de esta mezcla. Sin embargo, no todos los efectos de la contaminación fotoquímica pueden ser atribuidos al ozono. Así, algunas de las molestias causadas por la contaminación como, por ejemplo, la irritación ocular, lo son por compuestos tales como los nitratos orgánicos y los aldehidos ${ }^{32}$.

La comparación de los resultados de estudios experimentales y epidemiológicos sugiere que el ozono es la principal causa de los efectos de la contaminación fotoquímica sobre la salud en Norteamérica, sin embargo, en Europa, hasta fechas muy recientes, existían pocos estudios al respecto. A la luz de los mismos, en 1992, en un trabajo patrocinado por la oficina europea de la $\mathrm{OMS}^{19}$, se definieron los niveles en las concentraciones de efecto para los que se esperarían unos efectos agudos (tabla 4). Los síntomas y efectos sobre la función pulmonar causados por exposiciones a ozono de 1 hora a concentraciones de alrededor de $200 \mu \mathrm{g} / \mathrm{m}^{3}$, se clasificaron como leves. En el otro extremo de la escala, la combinación e intensidad de los efectos esperados por exposiciones a concentraciones de ozono de $400 \mu \mathrm{g} / \mathrm{m}^{3}$ y superiores se clasificaron como severas.

Estos niveles no indican que exista un umbral para el desencadenamiento de los efectos, sino que concentraciones como las indicadas son lo bastante altas como para causar efectos que pueden ser detectados por medio de estudios bien diseñados. Niveles más altos de exposición causarían efectos de mayor severidad, además de aumentar la fracción de población expuesta; sin embargo, esto no es posible de definir debido al limitado número de estudios realizados hasta el momento. Un nivel de contaminación más bajo que el considerado en la tabla 4 no presupone ausencia de efectos, pero, al menos, no se esperaría que causara problemas de salud importantes. En general, las personas que previamente padecen alguna enfermedad respiratoria o deficiencias del aparato circulatorio son más sensibles a sufrir trastornos de importancia, incluso con elevaciones de contaminación fotoquímica relativamente pequeñas ${ }^{14}$.

Tabla 4

Efectos agudos esperados por contaminación fotoquimica en días caracterizados por niveles máximos de concentraciones de ozono de 1 hora. Indicado para niños y adultos jóvenes no fumadores sobre la base de las observaciones hechas en estudios toxicológicos, clínicos y epidemiológicos

\begin{tabular}{|c|c|c|c|c|c|c|c|}
\hline \multirow[t]{2}{*}{$\begin{array}{l}\text { Nivel de } \\
\text { ozono } \\
\left(\mu g / m^{3}\right)\end{array}$} & \multirow[t]{2}{*}{$\begin{array}{l}\text { Irritación de ojos, } \\
\text { nariz y garganta }\end{array}$} & \multicolumn{2}{|c|}{$\begin{array}{c}\text { Media de disminucion de FEM } \\
\text { en poblacion activa en el } \\
\text { exterior }\end{array}$} & \multirow[t]{2}{*}{$\begin{array}{l}\text { Impedimento para } \\
\text { realizar activida- } \\
\text { des al exterior }\end{array}$} & \multirow[t]{2}{*}{$\begin{array}{l}\text { Respuesta inflamato- } \\
\text { ria, hiperreactividad } \\
\text { en personas activas } \\
\text { en el exterior }\end{array}$} & \multirow[t]{2}{*}{$\begin{array}{c}\text { Sintomas } \\
\text { respiratorios } \\
\text { (principalmente } \\
\text { en adultos) }\end{array}$} & \multirow[t]{2}{*}{$\begin{array}{l}\text { Clasificación } \\
\text { global }\end{array}$} \\
\hline & & $\begin{array}{l}\text { Población } \\
\text { general }\end{array}$ & $\begin{array}{l}10 \% \text { más } \\
\text { sensible de la } \\
\text { población }\end{array}$ & & & & \\
\hline$<100$ & Sin efecto & $0 \%$ & $0 \%$ & $0 \%$ & Ninguna & Ninguno & - \\
\hline 200 & $\begin{array}{l}\text { En unas pocas } \\
\text { personas sensibles }\end{array}$ & $5 \%$ & $10 \%$ & $0 \%$ & Leve & $\begin{array}{l}\text { Opresión en el } \\
\text { pecho, tos }\end{array}$ & Ligero \\
\hline 300 & $\begin{array}{c}<30 \% \\
\text { de la población }\end{array}$ & $15 \%$ & $30 \%$ & $\begin{array}{l}\text { Algunos } \\
\text { individuos }\end{array}$ & Moderada & $\begin{array}{l}\text { Incremento de } \\
\text { sintomas }\end{array}$ & Moderado \\
\hline 400 & $\begin{array}{c}>50 \% \\
\text { de la población }\end{array}$ & $50 \%$ & $50 \%$ & $\begin{array}{c}\text { Muchos } \\
\text { individuos }\end{array}$ & Severa & $\begin{array}{l}\text { Mayor incremen- } \\
\text { to de sintomas }\end{array}$ & Severo \\
\hline
\end{tabular}

Fuente: OMS, $1992^{19}$

FEM: Flujo expiratorio máximo 
En los últimos dos años varios estudios epidemiológicos han mostrado el efecto de los niveles de ozono sobre diferentes indicadores de actividad sanitaria. En Europa el proyecto APHEA ha publicado recientemente 3 meta-análisis sobre el efecto a corto plazo de la contaminación atmosférica en diversas ciudades europeas, en las que el ozono formaba parte de los contaminantes estudiados. Un incremento de $50 \mu \mathrm{g} / \mathrm{m} 3$ en los niveles de ozono se asoció con un aumento del $4 \%$ (IC ${ }_{95 \%} 2 \%$ a $7 \%$ ) en el número de ingresos hospitalarios por enfermedad pulmonar obstructiva crónica (EPOC) ${ }^{33}$, del $3,5 \%$ ( $\mathrm{IC}_{95 \%}-6 \%$ a $14 \%$ ) en los ingresos por asma en mayores de 15 años $^{34}$ y del $2,3 \%$ (IC ${ }_{95 \%} 1 \%$ a $4,9 \%$ ) en el número de defunciones por todas las causas ${ }^{35}$.

Además de los ingresos por enfermedades respiratorias y mortalidad, también se ha analizado la asociación del ozono con los ingresos por enfermedades cardiovasculares. En Canadá, Burnett et al ${ }^{36}$ analizan el papel de diversos contaminantes sobre los ingresos por insuficiencia cardiaca en 10 ciudades (sumando un total de 12,6 millones de habitantes). Un aumento en 25 $\mu \mathrm{g} / \mathrm{m} 3$ en los niveles de ozono se asoció de forma significativa con un incremento del $2,7 \%$ en el número de ingresos. Sin embargo, las estimaciones fueron consistentes solamente para el $\mathrm{CO}$. Otros estudios, realizados tanto en América del Norte ${ }^{37-40}$ como en Europa ${ }^{41}$, no han podido demostrar un efecto significativo del ozono sobre los ingresos por enfermedades del aparato circulatorio.

A nivel experimental, en un estudio reciente ${ }^{43}$ realizado en un grupo seleccionado de sujetos no se ha podido demostrar un efecto significativo sobre determinados parámetros hemodinámicos tras la exposición controlada a niveles moderados de ozono $(0,3 \mathrm{ppm})$, de manera que los posibles efectos del mismo sobre el sistema cardiovascular se producirían de forma indirecta, por medio de su acción sobre el árbol respiratorio.
Como conclusión, se puede afirmar que existc un amplio consenso en aceptar los efectos nocivos de la contaminación atmosférica en la salud. Sin embargo, este consenso no es tan amplio a la hora de establecer, entre otras cuestiones, qué niveles pueden ser considerados como seguros para el conjunto de la población, cuáles son los posibles efectos crónicos de la contaminación, qué compuestos tóxicos son los responsables del daño en salud así como los mecanismos fisiológicos de desencadenamiento del efecto. Todas ellas están siendo planteadas en el ámbito de diferentes proyectos de investigación, en especial proyectos multicéntricos, llevadas a cabo por especialistas de diversos campos, como la epidemiología, la toxicología, la clínica o la medición de la exposición.

\section{BIBLIOGRAFÍA}

1. Jendritzky G. The atmospheric environment. An introduction. Experientia 1993;49(9):733-40.

2. Eschenbacher W, IIolian A, Campion R. Air toxics and asthma: impacts and end points. Environ Health Perspect 1995;103 Suppl 6:209-11.

3. Ware J, Thibodeau L, Speizer F, Colome S, Ferris B. Assessment of the health effects of atmospheric sulphur oxides and particulate matter: evidence from observational studies. Environ Health Perspect 1981:41:255-76.

4. Pope C, Kanner R. Acute Effects of PM10 Pollution on Pulmonary Function of Smokers with Mild to Moderate Chronic Obstructive Pulmonary Disease. Am Rev Respir Dis 1993;147: 1336-40.

5. Touloumi G, Samoli E, Katsouyanni K. Daily mortality and «winter type» air pollution in Athens, Greece-a time series analysis within the APHEA project. J Epidemiol Community Health 1996;50 Suppl 1:s47-51.

6. A Committe of the Environmental and Occupational Health Assembly of the Amcrican Thoracic Society. Health effects of outdoor air pollution (Part 1). Am J Respir Crit Care Med 1996;153:3-50.

7. Folinsbee LJ. Human Health Effects of Air Pollution. Environ Health Perspect 1992;100:45-56. 
8. Williams, K., editor. Exposure assessment. COST 613/2 report series on air pollution epidemiology. Report Number 1. Brussels. Comission of the European Communities. DG XII, FUJR 14345 EN. 1992; 1.

9. Morgensten H. Ecologic studies in epidemiology: concepts, principles, and methods. Annu Rev Public Health 1995;16:61-81.

10. Sáez M, Pérez Hoyos S, Tobias A, Saurina C, Barceló M, Ballester F. Métodos de series temporales en los estudios epidemiológicos sobre contaminación atmosférica. Rev Esp Salud Pública 1999;73:133-143.

11. Modélisation des liens à court terme entre la pollution atmosphérique et la santé. Un exemple: $\mathrm{SO}_{2}$ et mortalité totale, Paris, 1987-1990. Rev Epidemiol Santé Publ 1998;46:316-28.

12. Goldsmith J, Friger M, Abramson M. Associations between health and air pollution in time-scries analyses. Arch Environ Health 1996;51(5):359-67.

13. Kalkstein L. A New Approach to Evaluate the Impact of Climate on Iluman Mortality. Environ Health Perspect 1991;96:145-50.

14. Dockery D, Pope C, Xu X, Spengler J, Ware J, Fay M, Ferris B, Speizer F. An association between air pollution and mortality in six U.S. cities [see comments]. N Engl J Med 1993;329(24): 1753-9.

15. Katsouyanni K, Schwartz J, Spix C, Touloumi G, Zmirou D, Zanobetti A, Wojtyniak B, Vonk J, Tobias A, Ponka A, et al. Short term effects of air pollution on health: a European approach using epidemiologic time series data: the APHEA protocol. J Epidemiol Commun Health 1996;50 Suppl 1:\$12-8.

16. APHEA2. Short-term effects of air pollution on health: a European approach to methodology, dose-response assessment and evaluation of public health significance. Study proposal. 1997.

17. Roemer W, Hoek G, Brunekreef B, Schouten J, Baldini G, Clench-Aas J, Englert N, Fischer P, Forsberg B, Halusszka $J$, et al. Effect of short-term changes in urban air pollution on the respiratory health of children with chronic respiratory symptoms - The PEACE project: Introduction. Eur Respir Rev 1998;8(52):4-11.

18. Bates D. Health Indices of the Adverse Effects of air Pollution The Question of Coherence. Environ Res 1992;59:336-49.

19. WHO, editor.Acute effects on health of smog episodes. WHO Regional Publications European Series n. ${ }^{\circ} 43$. Copenhagen: WHO; 1992.
20. World Heath Organization (WHO)., editor.Air quality guidelines for Europe. WHO Regional Publications European Series n. ${ }^{\circ} 23$. Copenhagen: 1987.

21. WHO. Impact on human health from air pollution in Europe. Copenhagen: WHO Regional Office for Europe; 1990.

22. Dockery D, Pope C. Acute respiratory effects of particulate air pollution. Annu Rev Public Health 1994;15:107-32.

23. Moolgavkar S, Luebeck E. A critical review of the evidence on particulate air pollution and mortality. Epidemiol 1996;7(4):420-8.

24. Hoek G, Forsberg B, Borowska M, Hlawiczka S, Vaskövi V, Welinder H, Branis M, Benes I, Kotesovec F, Hagen LO, et al. Wintertime $\mathrm{PM}_{10}$ and Black Smoke concentrations across Europe: results from the PEACE study. Atmospheric Environ $1997 ; 3609-22$.

25. Seaton A, MacNee W, Donaldson K, Godden D. Particulate air pollution and acute health effects [see comments]. Lancet 1995;345(8943):176-8.

26. Peters A, Döring A, Wichmann H, Koenig W. Increased plasma viscosity during an air pollution episode: a link to mortality? Lancet 1997;349: 1582-7.

27. Kaiser J. Showdown over clean air science. Science 1997;277:466-9.

28. Katsouyanni K, Touloumi G, Spix C, Schwartz J, Balducci F, Medina S, Rossi G, Wojtyniak B, Sunyer J, Bacharova L, et al. Short-term effects of ambient sulphur dioxide and particulate matter on mortality in 12 European cities: results from time series data from the APHEA project. Air Pollution and Health: a European Approach. BMJ 1997;314(7095):1658-63.

29. Chestnut L, Schwartz J, Savitz D, Burchfjel C Pulmonary function and ambient particulate matter: epidemiological evidence from NHANES Arch Environ Health 1991;(46):135-44.

30. Ackermann-Liebrich $U$, Leuenberger $P$, Schwartz J, Schindler C, Monn C, Bolognini G, Bongard J, Brändli $O$, Domenighetti $G$, Elsasser $\mathrm{S}$, et al. Lung Function and Long Term Exposurc to Air Pollutants in Switzerland. Am J Respir Crit Care Med 1997;155:122-9.

31. Pope C, Thun M, Namboodiri M, Dockery D, Evans J, Speizer F, Heath C. Particulate air pollution as a predictor of mortality in a prospective study of U.S. adults. Am J Respir Crit Care Med 1995;151(3 Pt 1):669-74. 
32. WHO, Air quality guidelines for Europe. WHO Regional Publications European Series n. 23. Copenhagen: WHO; 1987.

33. Anderson H, Spix C, Medina S, Schouten J, Castellsague J, Rossi G, Zmirou D, Touloumi G, Wojtyniak $B$, Ponka $\Lambda$, et al. $\Lambda$ ir pollution and daily admissions for chronic obstructive pulmonary disease in 6 European citics: results from the APHEA project. Eur Respir J 1997;10(5):1064-71.

34. Sunyer J, Spix C, Quénel P, Ponce de Leon A, Barumandzadeh T, Touloumi G, Bacharova L, Wojtyniak B, Vonk J, Bisanti L, et al. Urban air pollution and emergency admissions for asthma in four European cities: the APHEA Project. Tho$\operatorname{rax} 1997 ; 52: 760-5$.

35. Touloumi G, Katsouyanni K, Zmirou D, Schwartz J, Spix C, Ponce de Leon A, Tobias A, Quénel P, Rabczenko D, Bacharova L, et al. Short-term Effects of Ambient Oxidant Exposure on Mortality: A Combined Analysis within the APHEA Project. Am J Epidemiol 1997;146(2):177-85.

36. Burnett R, Dales R, Brook J, Raizenne M, Krewski D. Association between Ambient Carbon Monoxide Levels and Hospitalizations for Congestive Heart Failure in the Elderly in 10 Canadian Cities. Epidemiol 1996;8(2):162-7.

37. Schwartz J, Morris R. Air pollution and hospital admissions for cardiovascular disease in Detroit, Michigan. Am J Epidemiol 1995;142(1):23-35.
38. Morris R, Naumova F, Munasinghe R. Ambient Air Pollution and Hospitalization for Congestive Heart Failure among Elderly People in Seven Large US Cities. Am J Public Health 1995; 85(10):1361-5.

39. Schwartz J. Air Pollution and IIospital Admissions for Cardiovascular Disease in Tucson. Epidemiol 1997;8(4):371-7.

40. Burnett R, Cakmak S, Brook J, Krewski D. The role of particulate size and chemistry in the association between summertime ambient air pollution and hospitalization for cardiorespiratory diseases. Environ Health Perspect 1997;105: 614-20.

41. Ponka A, Virtanen M. Low-level air pollution and hospital admissions for cardiac and cerebrovascular diseases in Helsinki. Am J Public Health 1996;86(9):1273-80.

42. Andrews C, Buist S, Chaitman B, Hackney J, Rom W, Samet J, Schenker M, Shy C, Strieder D. Guidelines as to What Constitutes and Adverse Respiratory Health Effect, with Special Reference to Epidemiologic Studies of Air Pollution. Am Rev Respir Dis 1985;131:666-8.

43. Gong H, Wong R, Sarma RJ, Linn WS, Sullivan ED, Shamoo DA, et al. Cardiovascular effects of ozone exposure in human volunteers. Am J Respir Crit Care Med 1998; 158: 538-46. 